\title{
Does Social Support Really Help to Eat a Low-Fat Diet? Main Effects and Gender Differences of Received Social Support within the Health Action Process Approach
}

\author{
Urte Scholz* \\ University of Konstanz, Germany \\ Sibylle Ochsner and Rainer Hornung \\ University of Zurich, Switzerland \\ Nina Knoll \\ Freie Universität Berlin, Germany
}

\begin{abstract}
Background: Most theories of health-behavior change focus exclusively on individual self-regulation without taking social factors, such as social support, into account. This study's first aim was to systematically test the added value of received instrumental and emotional social support within the Health Action Process Approach (HAPA) in the context of dietary change. In the social support literature, gender effects emerge with regard to the effectiveness of social support. Thus, a second aim was the examination of gender differences in the association of social support with dietary behavior. Methods: Participants were 252 overweight and obese individuals. At baseline and 12 months later, participants completed questionnaires on HAPA variables; diet-specific received social support and low-fat diet. Results: For the prediction of intentions 12 months later, instrumental support was more beneficial for men than for women over and above individual self-regulation. In terms of dietary behavior at T2, a moderate main effect of instrumental support emerged. Moreover, received emotional social support was beneficial for men, but not for women in terms of a low-fat diet 12 months later. Conclusions: Effects of received instrumental social support found in this study provide new evidence for the added value of integrating social support into the HAPA.
\end{abstract}

Keywords: dietary behavior, gender, Health Action Process Approach, healthbehavior change, received social support

\footnotetext{
* Address for correspondence: Urte Scholz, University of Konstanz, Department of Psychology, Developmental \& Health Psychology, PO Box 14, 78457 Konstanz, Germany. Email: urte.scholz@uni-konstanz.de
} 


\section{INTRODUCTION}

Changing one's health behavior is a difficult endeavor. There are several theories and models that explain the process of intention formation and health-behavior change, for example the Theory of Planned Behavior (TPB; Ajzen, 1991) and Social-Cognitive Theory (SCT; Bandura, 2001). All these theories have in common that they focus almost exclusively on individual self-regulation, such as attitudes or self-efficacy as predictors of intentions or behavior. Social exchange processes, such as social support, are not integral parts of these models or are considered only indirectly. At the same time, there is a growing literature on the impact of social support on health behavior (e.g. Steptoe, Perkins-Porras, Rink, Hilton, \& Cappuccio, 2004). These studies in turn, usually do not take into account common theories and models of health behavior. This paper aims at integrating both lines of research and also takes potential gender differences in the effects of social support into account.

\section{Individual Self-Regulation in Health-Behavior Change}

The theoretical basis of the present study is the Health Action Process Approach (Schwarzer, 1992, 2008). This model expands the TPB or SCT, in that it does not specify intentions to be the most important predictor of behavior, but also takes volitional variables into account that are assumed to explain the translation of intentions into behavior. For intention formation, the HAPA assumes high risk awareness, high positive and low negative outcome expectancies, and high self-efficacy to be of importance. Risk awareness refers to the perceived risk of a health threat due to a certain behavior (Schwarzer, 2008). Outcome expectancies are perceived advantages (pros) and disadvantages (cons) of a behavioral change. Self-efficacy refers to the optimistic belief in one's ability to master difficult tasks effectively (Bandura, 2001).

After intentions have been formed, the intended behavior change needs to be implemented. Besides high intentions, the HAPA assumes high self-efficacy, high action planning, and high action control to be relevant for successfully changing one's behavior. Action planning refers to forming concrete plans on when, where, and how to implement the target behavior (Lange et al., 2013; Sniehotta, Schwarzer, Scholz, \& Schüz, 2005b). Action control comprises three subfacets: awareness of standards, self-monitoring, and self-regulatory effort (Sniehotta et al., 2005a). Moreover, received social support is specified in the HAPA as a potential opportunity or barrier in the volitional phase, but so far has rarely been included in empirical studies (e.g. Burkert, Scholz, Gralla, Roigas, \& Knoll, 2011). 
Overall, the HAPA has been demonstrated to be of good predictive validity with regard to different health behaviors and different populations (e.g. Koring et al., 2012; Schwarzer et al., 2007). Like most other models of health behavior, however, the empirical research on the HAPA almost exclusively focuses on individual self-regulation and only rarely accounts for direct social influences on intention formation and behavior. As human behavior usually takes place within a social context, social support might play an important role for health-behavior change over and above individual self-regulation. Thus, the present study aims at integrating social support into the HAPA in order to test whether social support adds to the prediction of intentions and behavior over and above individual self-regulation.

\section{Social Support and Health Behavior}

Received social support refers to retrospective reports of actual support transactions (Schwarzer \& Knoll, 2010). This is in contrast to perceived social support, which depends relatively little on actual support transactions and is known to be relatively stable (Knoll, Rieckmann, \& Kienle, 2007; Sarason, Sarason, \& Shearin, 1986). This study focuses on received social support (Sarason et al., 1986) because received support is a more precise indicator of what actually happened in terms of support interactions in a given time span than is perceived support. A functional distinction can be made between emotional and instrumental social support. Emotional social support refers to being comforted or encouraged when facing problems. Instrumental social support refers to practical help, such as receiving help with household chores (Schwarzer \& Knoll, 2010).

Most studies report positive associations between social support and dietary behavior (e.g. Hagler et al., 2007; Jackson, 2006; Thrasher, Campbell, \& Oates, 2004) or change in dietary behavior (e.g. Kelsey et al., 2000; Steptoe et al., 2004). However, most studies on the association between social support and health behavior assess perceived social support. Moreover, there are far more studies focusing on cross-sectional associations between support and behavior than on longitudinal associations. Furthermore, almost all studies assess a general measure of social support without explicitly differentiating between instrumental and emotional social support. Finally, the vast majority of studies on social support and health behavior do not take the existing theories of health-behavior change into account. In the context of dietary behavior, in particular, people mostly display the behavior within a social context, which might evoke social support transactions. Thus, in order to properly capture these support transactions and to test for their longer-term effects, this study focused on integrating received emotional and instrumental social support into an existing health-behavior change theory applying a longitudinal design. 


\section{Social Support and Existing Models of Health-Behavior Change}

As outlined above, the HAPA (Schwarzer, 1992) in its very first version already specified social support as a variable that might come into play in the volitional phase of behavior change. Yet very few studies, and none with regard to dietary behavior, have explicitly addressed the role of social support within the HAPA. Another popular model of behavior change that comprises a measure of social support is the Transtheoretical Model of Behavior Change (TTM; Prochaska \& DiClemente, 1983). In the TTM one of ten processes of change is "helping relationships" (Prochaska \& DiClemente, 1983). Helping relationships are defined as the support of others during behavioral change and is operationalised as a measure of perceived social support. Results of studies that focused on the effects of helping relationships are inconsistent. There are studies demonstrating the importance of helping relationships during the behavior-change process (Lowther, Mutrie, \& Scott, 2007). Other studies could not confirm the effectiveness of helping relationships for behavioral change in the TTM (Andersen, 2006).

With regard to other theories of health-behavior change (e.g. the TPB and SCT), so far there are only a few studies that have tried to integrate social support into these theories in order to systematically test whether social support contributes to intention formation and behavior change over and above individual self-regulation (Courneya, Plotnikoff, Hotz, \& Birkett, 2000; Hamilton \& White, 2008; Strating, van Schuur, \& Suurmeijer, 2006). In the context of dietary behavior, there are even fewer studies. For example a study by Povey, Conner, Sparks, James, and Shepherd (2000) tested the impact of perceived social support on healthy eating within the framework of the TPB. The authors found no main effects of perceived social support on intentions, but interactions with attitudes and perceived behavioral control. This study, however, did not test for the effects of perceived social support on behavior. Another study by Anderson, Winett, and Wojcik (2007) tested the effect of perceived family support on different indicators of a healthy diet within the framework of the SCT. Results of this study confirmed indirect (via self-efficacy and self-regulation) but no direct effects of perceived social support on indicators of healthy dietary behavior.

Thus, to the best of our knowledge, so far no study has tested the role of received social support for the prediction of intentions and dietary behavior in the context of the HAPA. This is an important gap for at least two reasons: First, as outlined above received and perceived social support are distinct constructs, with received social support being the measure of actual support transactions. Second, for the prediction of behavior the HAPA takes variables into account that come into play after intention formation. Thus, 
testing the effects of received social support on behavior within the HAPA provides an even stronger test than testing received social support only against intentions and self-efficacy.

\section{Gender Effects of Social Support}

Findings on the stress-buffering effects of received support indicate that women and men usually benefit more from support received from women than from men (Glynn, Christenfeld, \& Gerin, 1999; Neff \& Karney, 2005; Westmaas, Wild, \& Ferrence, 2002). These results apply to strangers (e.g. Glynn et al., 1999) as well as to couples (e.g. Neff \& Karney, 2005). It seems that the provider's gender makes all the difference. Explanations for these gender differences range from findings demonstrating that women seem to be better able to adapt their support provision to the changing needs of their husbands whereas men seem to react with more negativity to their wive's stress (Neff \& Karney, 2005). This is in line with research showing higher ability of empathic accuracy in females (Thomas \& Fletcher, 2003). From the studies on social support and dietary behavior there is also some evidence for gender differences in the effectiveness of social support. When perceived social support is measured, it seems that women but not men benefit from social support with regard to their dietary behavior (e.g. Jackson, 2006; Kelsey et al., 2000). Less is known about the gender effects of received social support on dietary behavior. Moreover, so far none of the studies that tested the effects of social support on intentions and behavior within an existing theory of health-behavior change has tested for potential differences between women and men.

\section{The Current Study}

The context of this study was change in fat consumption in overweight and obese individuals. Overweight and obesity are classified as epidemic diseases (World Health Organization, 2000). As increased body weight is related to higher morbidity and mortality, changing one's diet to a low-fat diet is recommended to most overweight and obese individuals (e.g. National Institutes of Health, 1998).

Changing one's dietary behavior can be difficult for several reasons. First, it might constitute a stressor, because for example people might feel hungry or they struggle with resisting temptations and get irritated. Thus, people might be in need of emotional social support in order to cope with these stressful experiences. And second, it might also be difficult for practical reasons (e.g. changing one's diet when the partner is not willing to also comply with the change). Thus, instrumental social support received from one's partner could be a helpful resource in the process of dietary change. 


\section{Aims of the Study}

The present study's aims were twofold: first, we aimed at testing the effects of received instrumental and emotional social support within the HAPA (Schwarzer, 2008) in order to provide a systematic test of whether social support is at all able to add to the prediction of intentions and dietary behavior across a 12-month period over and above self-regulation. We hypothesised that both kinds of received social support display an added value to the usual HAPA variable in predicting intentions and fat consumption. Second, this study aimed at testing whether the associations between social support and intentions and health behavior are different for men and women. We hypothesised that men benefited more from received social support than women as the source of support in our study was the participant's heterosexual partner.

\section{METHOD}

\section{Sample and Procedures}

This longitudinal study was part of a larger randomised controlled trial aiming at changing dietary behavior in overweight and obese individuals (Ochsner, Scholz, \& Hornung, 2013; Scholz, Ochsner, \& Luszczynska, in press). ${ }^{1}$ For the present study, only those individuals who were married or living in a committed partnership were included. Overall, two points of measurement were considered: a baseline assessment (Time 1, T1) and a follow-up that took place 12 months later (Time 2, T2). The study was advertised in newspapers and on different webpages that were related to nutrition issues. Inclusion criteria were a Body Mass Index over 25 and being 18 years or older. Exclusion criteria were participation in a professional weight-loss program (e.g. Weight Watchers) and insufficient comprehension of the German language. Baseline assessment took place in the lab. Each participant gave informed consent and received a personal code to ensure anonymity. Participation was voluntary, and after completion of the study participants received 50 Swiss Francs. All individuals were treated according

\footnotetext{
${ }^{1}$ Parts of the data analyzed in the present paper were also used in the work by Scholz et al. (in press) and concerned change in dietary intake and social-cognitive mediators after different kinds of intervention. In a second paper from this data set (Ochsner et al., 2013) the focus was on the role of different kinds of self-efficacy beliefs in the process of changing one's diet. However, although there is some overlap with the variables used in the present paper, this paper presents the investigation of a unique research question not yet covered by previous publications from this project. Moreover, a subsample (only individuals who were married or living in a committed partnership) was included in the present study.
} 
to the ethical guidelines of the Helsinki Declaration 2000. The follow-up questionnaire together with a stamped return envelope were sent to participants by ordinary mail.

\section{Sample Characteristics}

The sample consisted of 252 participants ( $n=171$ women, $67.9 \%$ ) with a mean age of $52.34(S D=11.56$, range $=20-78)$. The majority of participants were married $(n=225,89.3 \%), 13$ individuals $(5.2 \%)$ were divorced, $11(4.4 \%)$ were single and three (1.2\%) were widowed. All individuals, however, were in a committed relationship. Most participants reported having attended nine years of school $(n=156,61.9 \%)$. Mean Body Mass Index was 30.84 $(S D=4.33)$.

\section{Measures}

Means, standard deviations, and Cronbach's alphas are displayed in Table 1. Unless otherwise indicated, the response format was a 6-point Likert scale $(1=$ completely disagree to $6=$ completely agree $)$. Risk awareness, positive outcome expectancies, self-efficacy, action planning, action control, received instrumental and emotional social support, and social desirability were assessed at baseline. Intentions were assessed at baseline and at T2. Fat consumption as an indicator of dietary behavior was assessed at T2.

Risk awareness was assessed by four items assessing situation-outcome expectations (Scholz, Nagy, Goehner, Luszczynska, \& Kliegel, 2009) for

TABLE 1

Means, Standard Deviations, and Internal Consistency for all Constructs of the Study $(N=252)$

\begin{tabular}{|c|c|c|c|c|c|c|c|c|}
\hline & \multicolumn{4}{|c|}{ Baseline (T1) } & \multicolumn{4}{|c|}{ 12-month follow-up (T2) } \\
\hline & M & SD & Range & $\alpha$ & M & $\mathrm{SD}$ & Range & $\alpha$ \\
\hline Low-fat dietary intake & & & & & 4.28 & 1.13 & $1-6$ & .75 \\
\hline Risk awareness & 4.03 & 1.25 & $1-6$ & .82 & & & & \\
\hline Pros & 5.37 & .79 & $1-6$ & .78 & & & & \\
\hline Intentions & 5.36 & .67 & $1-6$ & .82 & 5.04 & .79 & $1-6$ & .86 \\
\hline Self-efficacy & 4.60 & .98 & $1-6$ & .83 & & & & \\
\hline Action planning & 4.35 & 1.34 & $1-6$ & .84 & & & & \\
\hline Action control & 3.39 & 1.17 & $1-6$ & .90 & & & & \\
\hline Emotional social support & 3.96 & 1.49 & $1-6$ & .85 & & & & \\
\hline Instrumental social support & 3.97 & 1.59 & $1-6$ & .86 & & & & \\
\hline Social desirability & 11.20 & 2.50 & $4-16$ & .64 & & & & \\
\hline
\end{tabular}


example: "If I keep my lifestyle the way it is, there is a high likelihood that I will develop severe health problems."

Positive outcome expectancies regarding behavior change were measured with five items (adapted from Scholz et al., 2009). The stem "If I eat a low-fat diet, ..." was followed by positive consequences, such as ". . . then I can lose weight".

Behavioral intentions were measured with six items (adapted from Scholz et al., 2009). For example "I intend to eat a low-fat diet (e.g. low-fat meat, cheese, etc.)."

Self-efficacy was assessed with three items (adapted from Scholz et al., 2009), for example: "I am confident that I will succeed in eating a low-fat diet."

Action planning was assessed with four items (Scholz et al., 2009). For example: "I have made a detailed plan regarding when (at what meals) to change my eating habits."

Action control consists of nine items (Scholz et al., 2009). The item stem "During the past four weeks ..." was followed by items like "... I closely monitored my eating behavior", "... I had my intentions to change my eating habits often on my mind", and "... I tried my best to act in accordance to my intentions".

Received social support: Two different kinds of received social support were assessed: emotional and instrumental social support. Items were taken from the Berlin Social Support Scales (BSSS; Schulz \& Schwarzer, 2003) and adapted to the special setting of changing one's diet. Received emotional social support was assessed with four items. Received instrumental social support was assessed with three items. Participants were instructed to think of their partner. Then they were asked how this person reacted to the participant during the past week. An example item for emotional social support is "This person comforted me when I was feeling bad because of my failing to comply with my intentions regarding my diet." An example item for instrumental support is "This person helped me to change my dietary habits."

Fat consumption: Fat consumption was measured with a three item-scale (Renner et al., 2008; Renner \& Schwarzer, 2005). Items were: (a) "I am aware of my caloric intake", (b) "I follow a low-fat diet", (c) "If I happen to eat cake or chocolate, I eat only a little bit." Higher scores indicate lower dietary fat intake. Evidence for the validity of this measure is derived by Renner and Schwarzer (2005) demonstrating confirming correlations with a food frequency measure. Moreover, the above-cited articles provide evidence for the predictive validity of this instrument.

Social desirability was assessed with the SDS-17 (Stoeber, 2001) and served as control variable. Social desirability was included because in overweight individuals a certain bias in reporting their dietary behavior might be present (e.g. Lof \& Forsum, 2004). Social desirability captures a general tendency to respond in a positively biased way. Thus, controlling for social desirability in 
our analyses might reduce reporting bias on the dietary outcome. It was assessed at Time 1 only. The answer format was dichotomous (0 no, 1 yes). Higher values indicated higher social desirability.

\section{Data Analyses}

Dropout analyses (reported below) revealed that the missing pattern was at best missing at random (MAR; Graham, 2009). To account for patterns of missing data, we employed expectation maximisation (EM), including all mechanisms of missingness in our imputation model (Graham, 2009). This means that the imputed values are estimated on the basis of the dropout mechanisms and that they are thus less biased than resulting values from listwise deletion. For all analyses, univariate and multivariate outliers were routinely screened and treated as suggested by Tabachnick and Fidell (2001).

Our main analyses were hierarchical regression analyses. For the prediction of intentions and fat consumption the control variable, social desirability, and sex were entered in a first step. In a second step, all social-cognitive predictors as specified in the HAPA were entered. Emotional and instrumental social support were entered in a third step. In the final step, the interaction terms of emotional / instrumental social support and sex were entered. For the examination of possible interaction effects with sex, emotional and instrumental support were mean-centered to avoid problems with multicollinearity (Cohen, Cohen, West, \& Aiken, 2003). For the same reason, sex was coded 0 (for women) and 1 (for men). Interaction terms were generated by multiplying the mean-centered support variables with sex. To test whether the regression slopes were significant, we ran simple slope analyses (Preacher, Curran, \& Bauer, 2006).

As participants were part of a randomised controlled trial (further details on the intervention are available from the authors upon request ${ }^{1}$ ), we investigated possible involvement of the intervention in our models first. Intervention groups were dummy coded and were controlled for within the main regression analyses. As inclusion of intervention factors into the regression models did not change our findings, respective analyses and intervention factors are not further reported here. Instead, intervention groups were collapsed and data from all $N=252$ participants were analyzed.

\section{RESULTS}

\section{Dropout Analyses}

Of the 252 participants at baseline, 190 (75.4\%) completed the one-year follow-up. In terms of social-cognitive variables of the HAPA and received emotional social support, no significant differences emerged between drop- 
outs and continuers. There was a significant difference between dropouts and continuers for the BMI $(M=32.21, S D=4.99$ for dropouts, $M=30.39$, $S D=4.01$ for continuers), for instrumental social support $(M=3.62$, $S D=1.63$ for dropouts, $M=4.09, S D=1.57$ for continuers), and for low-fat dietary intake $(M=3.15, S D=.93$ for dropouts, $M=3.56, S D=1.18$ for continuers). In terms of socio-demographic variables no systematic dropout occurred regarding sex, age, marital status, or education.

\section{Descriptives}

In Table 2, all correlations of the HAPA variables, emotional and instrumental social support, sex, and social desirability are displayed. The correlation between emotional and instrumental social support was moderately high, indicating that both constructs measure distinct aspects of received social support. In terms of the standard HAPA variables, bivariate correlations indicated that positive outcome expectancies and self-efficacy, but not risk awareness, were positively associated with intentions at Time 2. Moreover, baseline measures of intentions, self-efficacy, action planning, and action control were all significantly related to fat consumption at time 2 on a bivariate level. In terms of the control variable social desirability, small but significant bivariate associations emerged for fat consumption and intentions at Time 2. Thus, social desirability was controlled for in all analyses. In terms of gender differences, small negative correlations emerged between sex and low-fat dietary intake as well as intentions and action planning. This indicated that men were less likely to intend to engage in a low-fat diet, to plan a low-fat diet, and to actually engage in a low-fat diet at Time 2. Moreover, a positive association between sex and instrumental received support emerged indicating that men received more instrumental social support than women.

\section{Prediction of Intentions}

For the prediction of intentions at $\mathrm{T} 2$, social desirability and sex were entered in a first step, followed by risk awareness, positive outcome expectancies, and self-efficacy in a second step, emotional and instrumental social support in a third, and the interaction terms in a fourth step. As can be seen in Table 3, emotional social support was not able to significantly explain variance in intentions 12 months later over and above the effects of the HAPA-specific predictors. Instrumental social support displayed a small and 10 per centlevel significant effect. However, a significant, albeit small, interaction effect between instrumental social support and sex emerged. Overall, 21 per cent of variance was explained in intentions one year after baseline assessment. As displayed in Figure 1, women reported high intentions to eat a low-fat diet one year later almost independently of the instrumental support received 


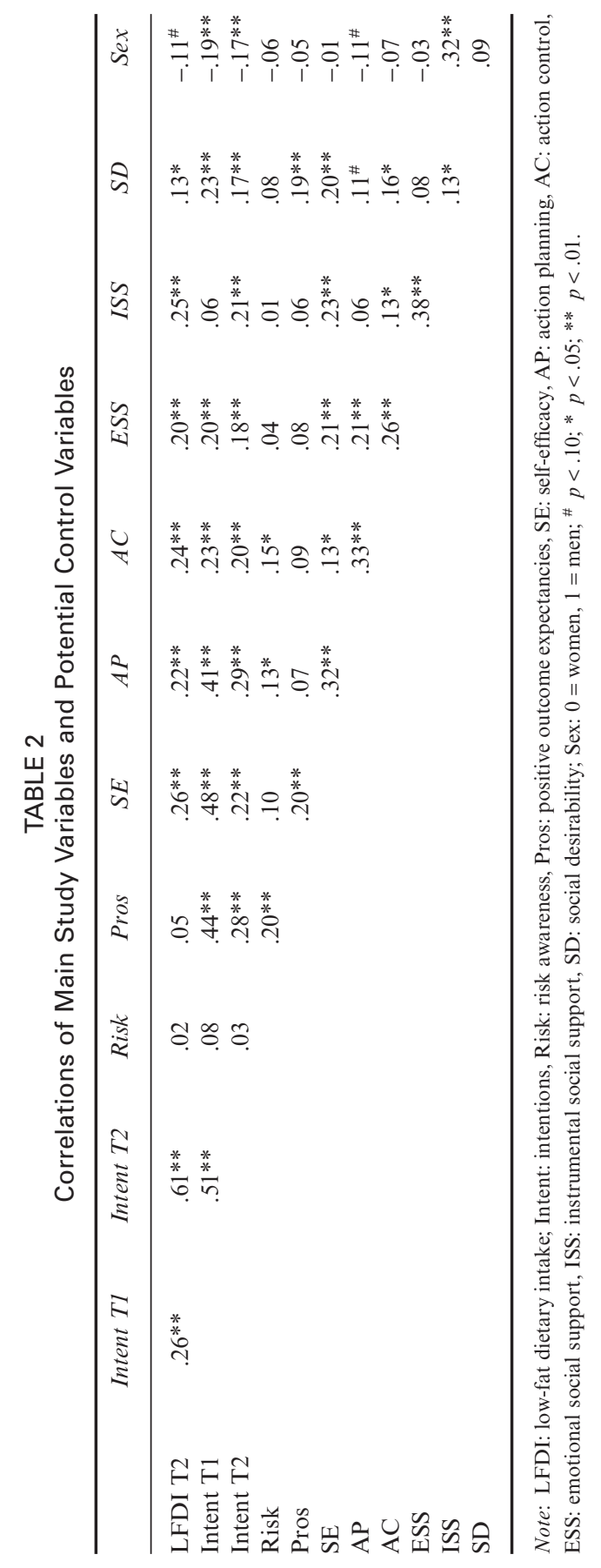


TABLE 3

Prediction of Intentions at T2 by HAPA Variables, and Emotional and Instrumental Social Support Moderated by Sex

\begin{tabular}{lccc}
\hline & \multicolumn{3}{c}{ Intentions at $T 2$} \\
\cline { 2 - 4 } Predictor & $\beta$ & $\mathrm{B}$ & $\mathrm{SE}$ B \\
\hline SD & .04 & .01 & .02 \\
Sex & $-.27^{* *}$ & -.45 & .11 \\
Risk & -.05 & -.03 & .04 \\
Pros & $.23^{* *}$ & .23 & .06 \\
SE & $.12^{*}$ & .10 & .05 \\
ESS & .03 & .02 & .04 \\
ISS & $.14^{\#}$ & .07 & .04 \\
ESS * sex & .05 & .05 & .07 \\
ISS * sex & $.17^{*}$ & .16 & .08 \\
$R^{2}$ total & .21 & & \\
Step 1 (SD, sex): $F(2,249)=6.75^{* *}, \Delta R^{2}=.05$ & & \\
Step 2 (risk, pros, SE): $F(3,246)=8.46^{* *}, \Delta R^{2}=.09$ & & \\
Step 3 (ESS, ISS): $F(2,244)=7.15^{* *}, \Delta R^{2}=.05$ & & \\
Step 4 (ESS*sex, ISS $* \operatorname{sex}): F(2,242)=3.30^{*}, \Delta R^{2}=.02$ & & \\
\hline
\end{tabular}

Note: Results: displayed are from the last step of hierarchical regression analysis. $\mathrm{T} 2=$ Time 2, 12 months later. SD: social desirability, Risk: risk awareness, Pros: positive outcome expectancies, SE: self-efficacy, ESS emotional social support, ISS: instrumental social support. Sex: $0=$ women, $1=$ men; ${ }^{\#} p<.10 ;{ }^{*} p<.05$; $* * p<.01$.

from their partner. For men, a different picture emerged: the more instrumental social support men received from their partner at T1, the higher their intentions to eat a low-fat diet 12 months later. Simple slope analyses confirmed that the association between instrumental social support and intentions at $\mathrm{T} 2$ was only marginally significant for women $(B=0.07 ; t(242)=1.7$, $p=.09)$, but significant for men $(B=0.23 ; t(242)=4.012, p=.001)$.

\section{Prediction of Fat Consumption}

For the prediction of low-fat dietary intake at $\mathrm{T} 2$, the predictive power of emotional and instrumental social support and their interaction with sex in competition with the HAPA variables sex and social desirability were examined. As can be seen in Table 4, instrumental social support displayed a significant positive effect over and above the control variables and the standard HAPA predictors. This indicates that the higher the received instrumental social support, the higher the reported low-fat dietary intake. Moreover, a significant interaction term between emotional social support and sex emerged. Overall, 22 per cent of variance was explained in low-fat dietary 


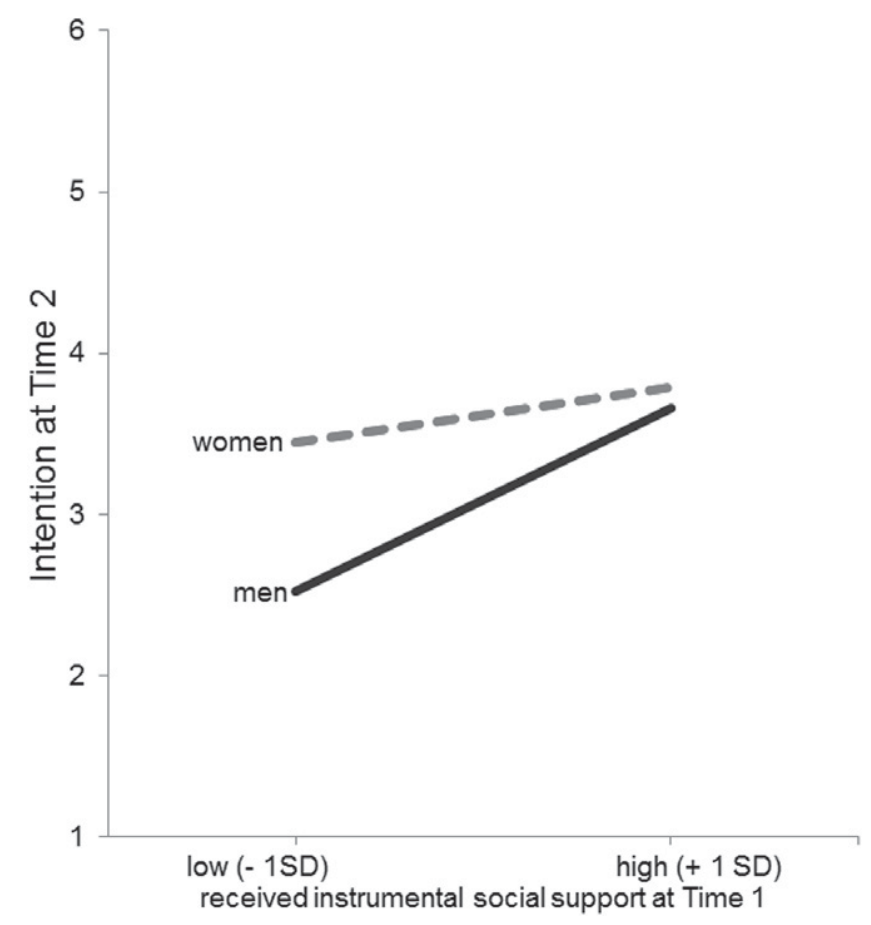

FIGURE 1. Interaction of instrumental social support and sex on intentions at Time 2.

intake one year after baseline assessment. The interaction is displayed in Figure 2: For women, the reported low-fat dietary intake one year later is high and independent of the received emotional support from their partners (simple slope analysis: $B=.10 ; t(242)=1.53, p=.13$ ). For men, however, a positive association emerged: the more emotional social support men received from their partners, the higher the reported low-fat dietary intake one year later (simple slope analyses: $B=.23 ; t(242)=2.64, p=.01$ ).

\section{DISCUSSION}

This study aimed at combining research from health-behavior change and social support by testing the effects of social support within an existing model of health-behavior change, the HAPA (Schwarzer, 2008), in a sample of overweight and obese individuals. This study was the first to differentiate between received emotional and instrumental social support in the context of dietary behavior and to test whether these effects of social support received 
TABLE 4

Prediction of Fat Consumption at T2 by HAPA Variables, and Emotional and Instrumental Social Support Moderated by Sex

\begin{tabular}{lccc}
\hline & \multicolumn{3}{c}{ LFDI at T2 } \\
\cline { 2 - 4 } Predictor & $\beta$ & $\mathrm{B}$ & SE B \\
\hline SD & .013 & .01 & .03 \\
Sex & $-.16^{*}$ & -.39 & .16 \\
Intent T1 & .11 & .18 & .12 \\
SE & .11 & .12 & .08 \\
AP & .06 & .05 & .06 \\
AC & $.12^{*}$ & .12 & .06 \\
ESS & -.12 & .19 & .06 \\
ISS & $.26^{* *}$ & .32 & .06 \\
ESS * sex & $.23^{* *}$ & .03 & .10 \\
ISS * sex & .02 & & \\
$R^{2}$ total & .22 & & \\
Step 1 (SD, sex): $F(2,249)=3.60^{*}, \Delta R^{2}=.03$ & & \\
Step 2 (AP, AC, SE, intent): $F(4,245)=7.74^{* *}, \Delta R^{2}=.11$ & \\
Step 3 (ESS, ISS): $F(2,243)=7.08^{* *}, \Delta R^{2}=.05$ & & \\
Step 4 (ESS*sex, ISS*sex): $F(2,241)=5.87^{* *}, \Delta R^{2}=.04$ & & \\
\hline
\end{tabular}

Note: Results displayed are from the last step of hierarchical regression analysis. T2 = Time 2, 12 months later. SD: social desirability, LFDI: low-fat dietary intake; Intent: intentions, SE: self-efficacy, AP: action planning, AC: action control, ESS: emotional social support, ISS: instrumental social support. Sex: $0=$ women, $1=$ men; ${ }^{\#} p<.10 ; * p<.05 ; * * \quad p<.01$

from one's partner on intentions and fat consumption differed between men and women. Results indicate that receiving instrumental social support from one's partner is beneficial for intending to eat a low-fat diet one year later for men, but not for women. One possible explanation for this gender effect is that in line with results from studies in the domain of social support and well-being (e.g. Glynn et al., 1999; Neff \& Karney, 2005) men and women benefit more from the support provided by women than by men. This is potentially due to the higher ability of women to correctly identify (Thomas \& Fletcher, 2003) and respond to another person's needs, whereas men seem to respond with more negativity to their partner's stress (Neff \& Karney, 2005). Another possible explanation for this moderation of gender is in line with a suggestion by Westmaas et al. (2002) who argue that caring for the health of one's partner or one's family members is inconsistent with the male gender role and might thus evoke negative reactions in female support recipients. More specifically in the domain of dietary behavior it might well be that in couples who follow a traditional role allocation (i.e. women buy groceries and prepare meals), the instrumental social support of women might be essential for forming and maintaining the intention to change one's diet in the 


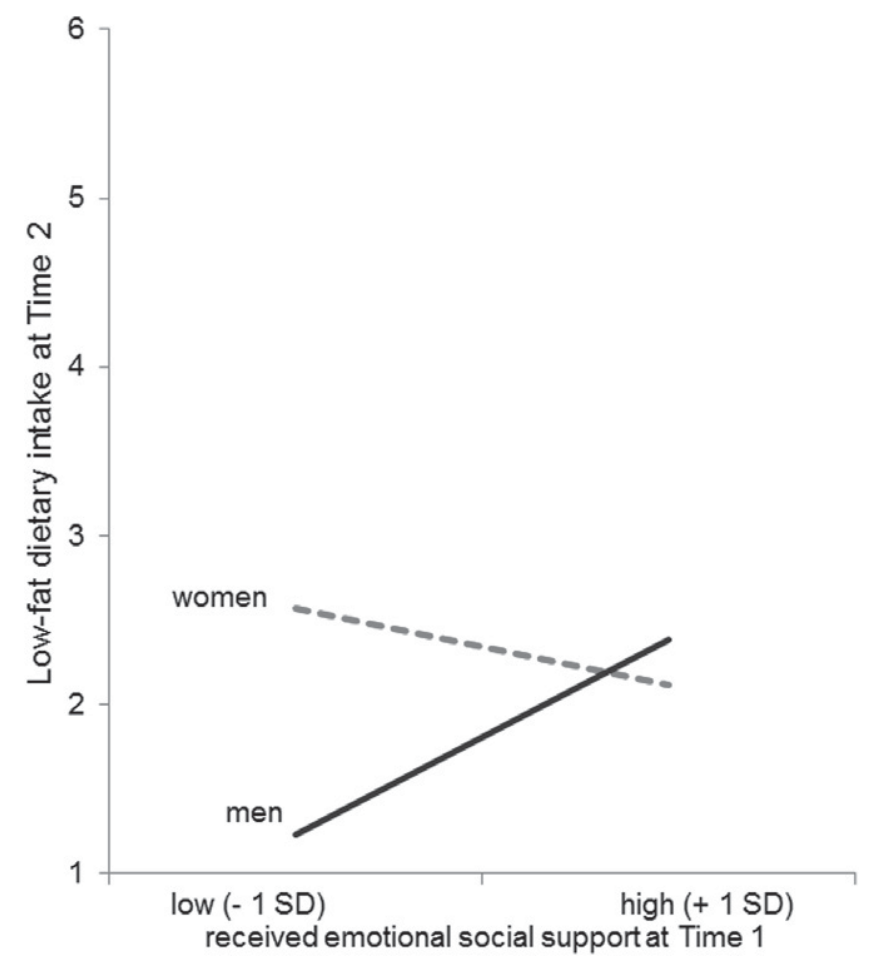

FIGURE 2. Interaction of emotional social support and sex on low-fat dietary intake at Time 2.

husbands whereas this might be less pronounced for the instrumental support provided by men. This might be different for other kinds of behavior (e.g. physical activity) that affect the other partner less. Thus, these results need to be replicated in other samples and across other behaviors. Moreover, results from studies that assessed perceived social support from different sources of support (e.g. significant others, family members, friends, etc.; Jackson, 2006) usually report opposite gender effects in that women benefit more from perceived social support than men. This emphasises not only the importance of distinguishing between received and perceived social support but also the fact that different sources of support (and more precisely the gender of these support sources) might make a difference for men and women.

The positive effects of receiving instrumental social support on behavior were not moderated by gender. Thus, instrumental social support received by one's partner seems to play an important role for a successful change in dietary behavior over and above a person's individual self-regulation 
competencies independent of the gender of the support receiver / provider. Thereby, although replication is clearly needed before final conclusions can be drawn, these results provide the first evidence that receiving instrumental social support might be considered an important predictor for change in specific behaviors to be integrated into the HAPA. The importance of instrumental social support in the context of changing one's diet is also emphasised by another effect found in this study: Individuals who reported receiving less diet-specific instrumental social support from their partner were more likely to drop out of the study. As we accounted for this systematic drop-out in the main analyses by applying appropriate missing imputation, this result becomes an interesting indicator of the potential impact of instrumental social support on changing one's diet per se. Any causal interpretation, however, is clearly not possible due to the correlational nature of our study.

In contrast to the consistent effects of instrumental support, receiving emotional support did not display any effects on intentions and no main effects on diet. This could indicate that overall the perceived stress of changing one's dietary behavior might have been rather low and that thus emotional social support was less relevant than instrumental social support. For the prediction of low-fat dietary intake, the effects of received emotional social support were moderated by gender in that again men in particular benefited from their partner's emotional support. Again, there are several potential explanations for this effect. First, as with instrumental social support, the sex of the provider of support might make a difference (e.g. Glynn et al., 1999; Neff \& Karney, 2005): When the stress of changing one's diet was experienced, emotional social support received from one's female partner might be more effective in easing this stress due to a higher correspondence between the recipient's needs and the type of support.

Effects associated with the individual self-regulation predictors of the HAPA were generally consistent with the assumptions of the HAPA, albeit rather small in effect size. An exception in terms of our expectations was the non-significant effects of action planning and self-efficacy on diet in the regression analyses. This was in contrast to the bivariate associations between action planning and self-efficacy with T2 low-fat dietary intake. Thus, one possible explanation for these non-significant effects in the regression analyses might be the shared variance of action planning, self-efficacy, and action control because action control remained the only significant predictor of T2 low-fat dietary intake. Action control can be considered the most proximal predictor of behavior compared to action planning and self-efficacy (Sniehotta et al., 2005a) and as a consequence might remain the only unique predictor.

Overall, explained variances of intentions and dietary behavior one year after baseline assessment were rather low. This is in part in contrast to other studies on dietary behavior applying a health-behavior change theory. For 
example, the study by Povey et al. (2000) explained over 57 per cent of variance in intentions and over 19 per cent of variance in behavior covering a one-month interval. Explained variance in behavior across a 6-week interval ranged between 35 per cent (fat consumption) and 61 per cent (fruit and vegetable consumption) in a study by Anderson et al. (2007). There are other studies, however, that also report lower levels of explained variance. For example, Steptoe and colleagues (2004) reported in a study across 12 months that none of the psychological variables measured at baseline contributed to explained variance of change in fruit and vegetable consumption, and social support measures were only able to explain 3 per cent of variance. Thus, given the time interval of 12 months in our study, explained variance is well within the documented range. Indeed compared to studies with a much shorter time interval (e.g. Anderson et al., 2007; Povey et al., 2000), the explained variance of intentions and behavior in our study can be considered substantial. Due to the design of the present study that focused on long-term effects in the outcomes but did not take into account changes in the predictors (e.g. Steptoe et al., 2004), we cannot exclude the possibility that baseline measures of individual self-regulation and social support might have changed considerably during the 12 months. As a consequence, future studies might want to adopt a mixture of macro- and micro-time perspectives in assessing daily or weekly measures of social-cognitive predictors and behavior during a certain time span in addition to long-term follow-ups. However, the small or even non-existent effects of the individual self-regulation variables again emphasise the importance of the effects of instrumental social support on behavior.

Another possible explanation for the low associations between predictor variables and criteria is a violation of the principle of compatibility recommended by Ajzen (1988). For example, risk awareness, action planning, action control, and social support do not refer to low-fat diet, but to eating habits or eating behavior which is on a more general level. Although, for example, gender displayed a substantial effect on intentions and on behavior despite being on a very different level of specificity, it can nonetheless be assumed that a higher compatibility in terms of levels of specificity of behavior would have led to stronger effects of the predictor variables. Future studies might thus want to optimise item wording in accordance with the principle of compatibility (Ajzen, 1988).

This study has several limitations. First, behavior was assessed by selfreport which may be subject to certain biases (Lof \& Forsum, 2004). For the assessment of dietary behavior, however, objective measures have their own disadvantages (e.g. impracticability, reactive changes in dietary behavior; see Wolper, Heshka, \& Heymsfield, 1995). Moreover, we controlled for social desirability in order to account for potential bias. Also, assessment of low-fat dietary behavior could be refined by applying a validated food frequency questionnaire (FFQ). The measure used in this study, however, was validated 
with a food frequency questionnaire and thus displays a good alternative to the sometimes quite burdening FFQs for participants. A second limitation was the systematic attrition present in this study. As outlined above, however, we accounted for this by appropriate missing data imputation (Graham, 2009).

Theoretical implications can be drawn from results of this study. For a conceptual expansion of the HAPA by received instrumental social support, this study needs replication with different samples and different behaviors. Moreover, the aim of the present study was to provide a basic test of the added value of received social support within the HAPA. Future studies might want to go a step further and test for interactions with the individual self-regulation variables (e.g. Povey et al., 2000). It might be possible that receiving social support might not only display main effects on intentions or behavior, but might also serve a compensatory role by benefiting those individuals whose self-regulatory competencies are underdeveloped. Finally, expanding the HAPA by received social support might contradict the principle of parsimony (Kerlinger, 1979). However, as results from this study emphasise, received social support as an indicator of social influences on health behaviors differs conceptually from the individual self-regulation variables of the model and might thus substantially add to our understanding of health-behavior change within a theoretical framework.

\section{ACKNOWLEDGEMENTS}

This study was funded in part by the Suzanne and Hans Biäsch Foundation for the Promotion of Applied Psychology. We would like to thank all student co-workers and interns who helped with data collection. While preparing this manuscript, the first author was partly funded (PP00P1_133632/1) and the second author was fully funded (100014_124516) by the Swiss National Science Foundation.

\section{REFERENCES}

Ajzen, I. (1988). Attitudes, personality, and behavior. Chicago, IL: Dorsey.

Ajzen, I. (1991). The Theory of Planned Behavior. Organizational Behavior and Human Decision Processes, 50(2), 179-211.

Andersen, S. (2006). Do indigenous helpers foster smoking cessation in adult smokers? Addictive Behaviors, 31(8), 1496-1502. doi: 10.1016/j.addbeh. 2005.10.008

Anderson, E.S., Winett, R.A., \& Wojcik, J.R. (2007). Self-regulation, self-efficacy, outcome expectations, and social support: Social cognitive theory and nutrition behavior. Annals of Behavioral Medicine, 34(3), 304-312.

Bandura, A. (2001). Social cognitive theory: An agentic perspective. Annual Review of Psychology, 52, 1-26. 
Burkert, S., Scholz, U., Gralla, O., Roigas, J., \& Knoll, N. (2011). Dyadic planning of health-behavior change after prostatectomy: A randomized-controlled planning intervention. Social Science \& Medicine, 73(5), 783-792. doi: 10.1016/j.socscimed. 2011.06.016

Cohen, J., Cohen, P., West, S.G., \& Aiken, L.S. (2003). Applied multiple regression / correlation analysis for the behavioral sciences (3rd edn.). Mahwah, NJ: Lawrence Erlbaum Associates.

Courneya, K.S., Plotnikoff, R.C., Hotz, S.B., \& Birkett, N.J. (2000). Social support and the Theory of Planned Behavior in the exercise domain. American Journal of Health Behavior, 24(4), 300-308.

Glynn, L.M., Christenfeld, N., \& Gerin, W. (1999). Gender, social support, and cardiovascular responses to stress. Psychosomatic Medicine, 61(2), 234 242.

Graham, J.W. (2009). Missing data analysis: Making it work in the real world. Annual Review of Psychology, 60, 549-576. doi: 10.1146/annurev.psych.58.110405.085530

Hagler, A.S., Norman, G.J., Zabinski, M.F., Sallis, J.F., Calfas, K.J., \& Patrick, K. (2007). Psychosocial correlates of dietary intake among overweight and obese men. American Journal of Health Behavior, 31(1), 3-12.

Hamilton, K., \& White, K.M. (2008). Extending the Theory of Planned Behavior: The role of self and social influences in predicting adolescent regular moderate-tovigorous physical activity. Journal of Sport and Exercise Psychology, 30(1), 5674.

Jackson, T. (2006). Relationships between perceived close social support and health practices within community samples of American women and men. Journal of Psychology, 140(3), 229-246.

Kelsey, K.S., Campbell, M.K., Tessaro, I., Benedict, S., Belton, L., Fernandez, L.M. et al. (2000). Social support and health behaviors among blue-collar women workers. American Journal of Health Behavior, 24(6), 434-443.

Kerlinger, F.N. (1979). Behavioral research: A conceptual approach. New York: Holt.

Knoll, N., Rieckmann, N., \& Kienle, R. (2007). The other way around: Does health predict perceived support? Anxiety, Stress and Coping, 20(1), 3-16. doi: 10.1080/ 10615800601032823

Koring, M., Richert, J., Parschau, L., Ernsting, A., Lippke, S., \& Schwarzer, R. (2012). A combined planning and self-efficacy intervention to promote physical activity: A multiple mediation analysis. Psychology, Health \& Medicine, 17(4), 488-498. doi: 10.1080/13548506.2011.608809

Lange, D., Richert, J., Koring, M., Knoll, N., Schwarzer, R., \& Lippke, S. (2013). Self-regulation prompts can increase fruit consumption: A one-hour randomised controlled online trial. Psychology \& Health. doi: 10.1080/08870446.2012.751107

Lof, M., \& Forsum, E. (2004). Validation of energy intake by dietary recall against different methods to assess energy expenditure. Journal of Human Nutrition and Dietetics, 17(5), 471-480. doi: 10.1111/j.1365-277X.2004.00554.x

Lowther, M., Mutrie, N., \& Scott, E.M. (2007). Identifying key processes of exercise behaviour change associated with movement through the stages of exercise behaviour change. Journal of Health Psychology, 12(2), 261-272. doi: 10.1177/ 1359105307074253 
National Institutes of Health (1998). Clinical guidelines on the identification, evaluation, and treatment of overweight and obesity in adults. NIH publication no. 98-4083.

Neff, L.A., \& Karney, B.R. (2005). Gender differences in social support: A question of skill or responsiveness? Journal of Personality and Social Psychology, 88(1), 79-90. doi: 10.1037/0022-3514.88.1.79

Ochsner, S., Scholz, U., \& Hornung, R. (2013). Testing phase-specific self-efficacy beliefs in the context of dietary behavior change. Applied Psychology: Health and Well-Being, 5(1), 99-117. doi: 10.1111/j.1758-0854.2012.01079.x

Povey, R., Conner, M., Sparks, P., James, R., \& Shepherd, R. (2000). The Theory of Planned Behaviour and healthy eating: Examining additive and moderating effects of social influence variables. Psychology \& Health, 14(6), 991-1006. doi: 10.1080/ 08870440008407363

Preacher, K.J., Curran, P.J., \& Bauer, D.J. (2006). Computational tools for probing interaction effects in multiple linear regression, multilevel modeling, and latent curve analysis. Journal of Educational and Behavioral Statistics, 31, 437-448.

Prochaska, J.O., \& DiClemente, C.C. (1983). Stages and processes of self-change of smoking: Toward an integrative model of change. Journal of Consulting and Clinical Psychology, 51(3), 390-395.

Renner, B., Kwon, S., Yang, B.H., Paik, K.C., Kim, S.H., Roh, S. et al. (2008). Social-cognitive predictors of dietary behaviors in South Korean men and women. International Journal of Behavioral Medicine, 15(1), 4-13. doi: 10.1080/ 10705500701783785

Renner, B., \& Schwarzer, R. (2005). The motivation to eat a healthy diet: How intenders and nonintenders differ in terms of risk perception, outcome expectancies, self-efficacy, and nutrition behavior. Polish Psychological Bulletin, 36, 715 .

Sarason, I.G., Sarason, B.R., \& Shearin, E.N. (1986). Social support as an individual difference variable: Its stability, origins, and relational aspects. Journal of Personality and Social Psychology, 50(4), 845-855.

Scholz, U., Nagy, G., Goehner, W., Luszczynska, A., \& Kliegel, M. (2009). Changes in self-regulatory cognitions as predictors of changes in smoking and nutrition behaviour. Psychology \& Health, 24(5), 545-561. doi: 10.1080/08870440801902519

Scholz, U., Ochsner, S., \& Luszczynska, A. (in press). Comparing different boosters of planning interventions on changes in fat consumption in overweight and obese individuals: A randomized controlled trial. International Journal of Psychology. doi: 10.1080/00207594.2012.661061

Schulz, U., \& Schwarzer, R. (2003). Social support in coping with illness: The Berlin Social Support Scales (BSSS). Diagnostica, 49(2), 73-82. doi: 10.1026//00121924.49.2.73

Schwarzer, R. (1992). Self-efficacy in the adoption and maintenance of health behaviors: Theoretical approaches and a new model. In R. Schwarzer (Ed.), Self-efficacy: Thought control of action (pp. 217-242). Washington, DC: Hemisphere.

Schwarzer, R. (2008). Modeling health behavior change: How to predict and modify the adoption and maintenance of health behaviors. Applied Psychology: An International Review, 57(1), 1-29. doi: 10.1111/j.1464-0597.2007.00325.x 
Schwarzer, R., \& Knoll, N. (2010). Social support. In J.W.A. Kaptein (Ed.), Health psychology (pp. 283-293). Oxford: Blackwell.

Schwarzer, R., Schüz, B., Ziegelmann, J.P., Lippke, S., Luszczynska, A., \& Scholz, U. (2007). Adoption and maintenance of four health behaviors: Theory-guided longitudinal studies on dental flossing, seat belt use, dietary behavior, and physical activity. Annals of Behavioral Medicine, 33(2), 156-166. doi: 10.1080/ 08836610701308221

Sniehotta, F.F., Scholz, U., Schwarzer, R., Fuhrmann, B., Kiwus, U., \& Völler, H. (2005a). Long-term effects of two psychological interventions on physical exercise and self-regulation following coronary rehabilitation. International Journal of Behavioral Medicine, 12(4), 244-255.

Sniehotta, F.F., Schwarzer, R., Scholz, U., \& Schüz, B. (2005b). Action planning and coping planning for long-term lifestyle change: Theory and assessment. European Journal of Social Psychology, 35(4), 565-576. doi: 10.1002/ejsp.258

Steptoe, A., Perkins-Porras, L., Rink, E., Hilton, S., \& Cappuccio, F.P. (2004). Psychological and social predictors of changes in fruit and vegetable consumption over 12 months following behavioral and nutrition education counseling. Health Psychology, 23(6), 574-581. doi: 10.1037/0278-6133.23.6.574

Stoeber, J. (2001). The Social Desirability Scale-17 (SDS-17): Convergent validity, discriminant validity, and relationship with age. European Journal of Psychological Assessment, 17(3), 222-232. doi: 10.1027//1015-5759.17.3.222

Strating, M.M.H., van Schuur, W.H., \& Suurmeijer, T.P.B.M. (2006). Contribution of partner support in self-management of rheumatoid arthritis patients: An application of the Theory of Planned Behavior. Journal of Behavioral Medicine, 29(1), 51-60. doi: 10.1007/s10865-005-9032-5

Tabachnick, B.G., \& Fidell, L.S. (2001). Using multivariate statistics (4th edn.). Boston, MA: Allyn and Bacon.

Thomas, G., \& Fletcher, G.J. (2003). Mind-reading accuracy in intimate relationships: Assessing the roles of the relationship, the target, and the judge. Journal of Personality and Social Psychology, 85(6), 1079-1094. doi: 10.1037/00223514.85.6.1079

Thrasher, J.F., Campbell, M.K., \& Oates, V. (2004). Behavior-specific social support for healthy behaviors among African American church members: Applying optimal matching theory. Health Education and Behavior, 31(2), 193-205. doi: $10.1177 / 1090198103259184$

Westmaas, J.L., Wild, T.C., \& Ferrence, R. (2002). Effects of gender in social control of smoking cessation. Health Psychology, 21(4), 368-376.

Wolper, C., Heshka, S., \& Heymsfield, S.B. (1995). Measuring food intake: An overview. In D.B. Allison (Ed.), Handbook of assessment methods for eating behaviors and weight related problems (pp. 215-240). London: Sage Publications.

World Health Organization (2000). Obesity: Preventing and managing the global epidemic. Report of a WHO consultation. Geneva: WHO. 